\title{
Aerodynamic flow simulation
}

\author{
R. W. Derksen ${ }^{1} \&$ J. Rimmer ${ }^{2}$ \\ ${ }^{I}$ Department of Mechanical and Manufacturing Engineering, \\ University of Manitoba, Winnipeg, Manitoba, Canada \\ ${ }^{2}$ E.H. Price, Winnipeg, Manitoba, Canada
}

\begin{abstract}
This paper reports our experience of applying a vortex cloud model to simulate the flow over airfoil sections at low-Reynolds numbers. Low-Reynolds number aerodynamics has become increasingly important of late due to interest in the development of unmanned aerial vehicles. The current state-of-the-art consists of a good base of modern experimental data, but relies on simulation methods based on high-Reynolds number experience. Vortex cloud models are numerical flow simulation methods that are based on inviscid flow tools. The method continuously injects many free vortices within the flow field and tracks their convection with time. The convective velocity is determined from the inviscid velocity component due to any bodies within the flow field, all free vortices, and a random component. The random component of the velocity field introduces a viscous effect and its value is scaled to the Reynolds number. Vortex cloud models are believed to be capable of modelling viscous flows and should be able to model separated flow without introducing special methods. We will provide an assessment of the predicted flow for a range of angles of attack for a selected set of airfoils when compared to the very good low-Reynolds number airfoil data compiled by Selig and his co-workers.
\end{abstract}

Keywords: aerodynamics, vortex cloud models, panel methods.

\section{Introduction}

The development of aerodynamic flow simulation methods has a substantial history that demonstrates a creative intellectual effort to overcome the difficulties inherent to the governing equations of fluid mechanics. Early efforts at simulating aerodynamic flows were limited by our inability to obtain analytical solutions to the Navier-Stokes equations to all but a few idealized examples. 
This resulted in an overwhelming reliance on experimental methods to obtain aircraft design information that has persisted up until quite recently. Aerodynamicists have developed a rich and interesting set of methods to obtain the approximate behaviour of aerodynamic structures that has evolved and become quite sophisticated. The first methods were based on the observation that inviscid flow methods could be used to model flow over streamline bodies at high Reynolds numbers. This allowed us to obtain reasonably accurate estimates of lift, pitching moments and induced drag using relatively simple analytical or computational methods. These methods cannot be used to estimate the viscous drag and, while very useful, are inadequate. A great deal of effort has been devoted to approximating viscous effects through the use of Prandtl's boundary layer theory. These efforts started by assuming that the airfoil was an equivalent flat plate, which is surprisingly accurate in estimating minimum drag, and were rapidly enhanced to include effect of the velocity distribution on the body. This approach was of very limited use and cannot be used over a sufficiently wide range of angle of attack due to flow separation. It was soon recognized that the flow over an airfoil could be more accurately approximated by iteratively employing an inviscid flow model with a boundary model. This method either creates a set of pseudo-bodies or a surface transpiration distribution that ultimately converges to a limiting state. Most methods are based on some variation of this approach at present. The deficiency of these methods comes from the ad hoc nature of how to deal with separation. The development of modern, high-speed, inexpensive computers has resulted in a number of fast and effective computational fluid dynamics (CFD) methods to solve viscous flows in many complex geometrical configurations, and have been applied to aerodynamic applications. These methods are based on the numerical solution of Navier-Stokes equations and should have no difficulty with flow separation. This approach is very time intensive and expensive and unfortunately has its own concerns.

The objective of this work was to examine a relatively simple, novel, numerical method, the vortex cloud model, as a tool for aerodynamic flow simulation. The developers of this method have suggested that it is suitable to simulate viscous flows in complex geometries and is capable of dealing with flow separation.

\section{The Vortex Cloud Model}

The Vortex Cloud Model was originally proposed by Chorin [1, 2] as a method to numerically solve viscous flows. It is based on the vorticity equation, derived from the Navier-Stokes equations, and is given by

$$
\frac{\partial \bar{\omega}}{\partial t}+(\bar{V} \cdot \nabla) \bar{\omega}=(\bar{\omega} \cdot \nabla) \bar{V}+v \nabla^{2} \bar{\omega} .
$$

This approach allows us to set aside the issue of the pressure field until the velocity field has been determined. We should note that many traditional 
numerical field solution methods (finite difference methods, etc.) for solving fluid flows have been based on a stream function - vorticity approach. The novelty of the vortex cloud method comes from its approach to solving the vorticity equation.

The vortex cloud method is based on simulating the motion of a cloud of discrete vortices that are continuously released into the flow field. The production of new vorticity is based on the surface vorticity distribution on any bodies within the flow field as obtained from an inviscid flow calculation, and the convection of the vorticity cloud is determined by the inviscid, induced velocities from the bodies and all other vortex elements, and a random component that depends on Reynolds number. The effect of the random component is to introduce a 'diffusivity' that is not present in ideal flows, and is believed to simulate the effect of viscosity. There are several advantages of the cloud model compared to a traditional field method. The first is that the flow field does not need to be discretized, as the location of each vortex is associated with the vortex. Secondly, the core calculations for the vortex cloud model are very simple and rapidly computed. Finally, the vortex cloud model should be able to more naturally deal with any separation regions that develop in the flow field.

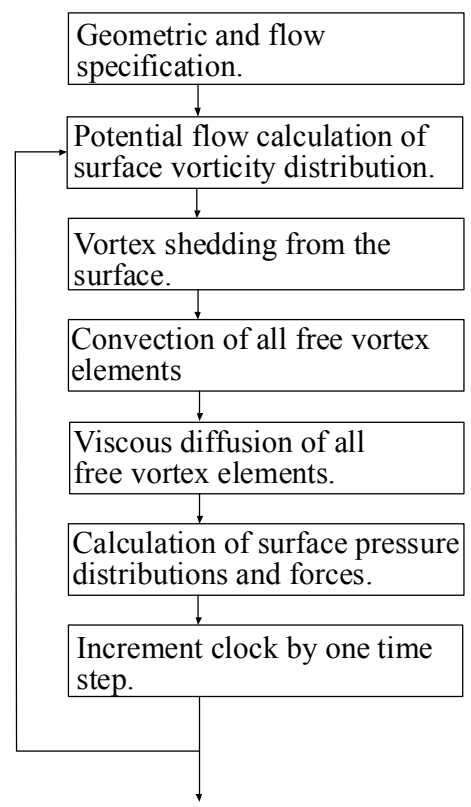

Figure 1: The basic vortex cloud method flow chart.

The basic steps of a vortex cloud model are shown in Figure 1. As to be expected the first step is to define the geometry of the airfoil and the required flow parameters. The initial step is to apply a vortex panel method to obtain the inviscid flow field about an airfoil in the absence of any free vorticity in the flow 
field. This gives an initial surface vorticity distribution which is used to shed a finite number of free vortices from the body into the flow. The position of the shed vortices is then advanced one time step using the induced velocities from the inviscid flow field. Viscous diffusion is then simulated by displacing the shed vortices by either randomly displacement of their position or applying a random velocity component. At this point, the instantaneous surface pressure distribution and aerodynamic forces are computed and accumulated for subsequent averaging. This process is repeated from the potential flow step, now including the influence of the shed vortices, till the simulation time or the average values are stable.

The key questions that need answering if the vortex cloud model is to be a successful method are:

1. How many individual vortices must be examined to obtain a sufficiently accurate flow field simulation?

2. How should we determine the production of vorticity, and are the existing methods satisfactory?

3. Can a suitable model for the random velocity components be developed, and if so do the existing models produce satisfactory results?

4. How many time steps are required to obtain stable average values of field variables such as the lift and drag on an object?

5. Is the simulation capable of modelling the unsteady components of the flow?

Several variations of vortex cloud models have been proposed and developed over the last 30 years. We have based our work on the method developed by R.I. Lewis and his co-workers [3].

\section{Code development}

The first step in coding this simulation was to develop a vortex panel method to compute the ideal flow over a cylindrical profile. The method divided the profile into $N$ linear panels with a uniform vorticity distribution, and results in an $N \times N$ linear system of equations. The panel lengths were adjusted to have a cosine distribution that clusters nodes near the leading and trailing edge of the profile. This reduces the error due to panels being close to each other at the trailing edge and provides greater detail at the leading edge where the flow changes rapidly. This code was then carefully examined by carefully examining its result compared to known analytical solutions. The vortex cloud code was developed from this base by adjusting the right hand side of the panel methods to include the effect of a distribution of free vortices in the flow field. This modification assumed that the location of each free vortex is known and that it has a given strength. Each call returned the surface velocity distribution and allowed us to calculate the induced velocity at any point in the flow field.

This was used by a modified Euler scheme to advance the location of all of the free vortices for a given time step. This process is faster than it may appear as the resulting system of equations does not depend on the location of the free vortices so only one matrix inversion needs to be done, so a velocity calculation 
is the result of a simple set of matrix multiplications. This does require a significant computational load over the Euler method but is necessary to minimize the vortex drift error to a tolerable level. We found no need to apply a higher order integration scheme as a time step that resulted in a sufficiently accurate prediction could be based on the free stream velocity and chord length. The performance of this code was compared the theoretical location of a single free vortex with its predicted location and found to be good.

At this point code was developed to insert a number of free vortices into the flow field. Each vortex was shed from the surface at the normal centre of a randomly selected panel a distance $10 \sqrt{v d t / 3}$. The shed vortex strength was set to $25 \%$ of the net panel vorticity, and panel vorticity adjusted to $75 \%$ of its previous value. The full panel vorticity could not be shed as it would result in instability in the simulation. The number of vortices created each time step was held a constant rate until the total number of vortices equalled a set maximum. Vortices were removed from the analysis for two reasons. First, when a vortex had moved more than four chord lengths behind the cylinder it was removed from the field and a new vortex was inserted. Secondly, vortices are also removed from the simulation if they cross into the body during the intermediate step in the modified Euler method and an additional free vortex was shed.

After the convection step, viscous diffusion was modelled by adding a random, two dimensional displacement to each of the free vortices. The scheme to determine these components was due to Lewis [3]. First two real values, $L$ and $K$ are set to random values and modified to

$$
L^{\prime}=2 \pi L
$$

and

$$
K^{\prime}=\sqrt{4 v d t \ln (1 / k)}
$$

Then $K^{\prime} \cos \left(L^{\prime}\right)$ was added to the vortex position's $x$-component and $K^{\prime} \sin \left(L^{\prime}\right)$ to the $y$-component. We should note that this hides the role of Reynolds number as $d t$ is proportional to the product of the cylinder chord length and free stream velocity.

An initial effort was made to compute the lift and drag by integrating the surface pressure distribution over the airfoil. This results in an instantaneous estimate of these quantities that must be averaged over a suitable number of time steps. Unfortunately, this integration has proven to be unstable and alternate means of estimating the lift and drag are being examined.

\section{Test cases}

The test case chosen was based on the availability of high quality experimental data. This naturally led to the outstanding work done by Selig and his coworkers $[4,5,6$, and 7] in the mid-nineties. This work details the results of a 
carefully constructed experimental program to produce a database of modern, low-Reynolds number airfoils. The measurements were exhaustively examined for their accuracy, for example drag was measured by the more accurate wake traverse method. This work filled a gap in previously existing databases by focusing in on low-Reynolds number, and is of great use in the design of $\mathrm{R} / \mathrm{C}$ aircraft and small unmanned aerial vehicles. We believe that this work sets a new standard on airfoil data.

Our initial work will focus on a single airfoil, the NACA 2414 airfoil shown in figure 2, and was selected for this study as a complete data set could be found in [6]. The data specified the ideal profile shape and the deviation of the experimental model from the ideal. The average difference for this particular airfoil was 0.0044 in.; Surface velocity distributions were given for incidences between -2 and $10^{\circ}$ at a Reynolds number of 200,000. Additionally, lift curves and drag polars were given for Reynolds numbers of 60,000, 100,000, 200,000, and 300,000. Future work will examine a larger number of cases from this database.

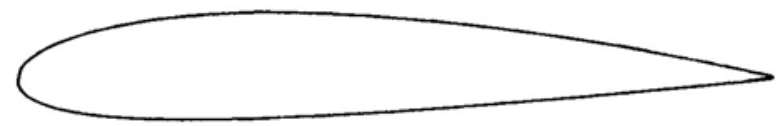

Figure 2: $\quad$ Airfoil profile for a NACA 2412 section.

The data indicated a nearly linear lift curve up to an angle of incidence of roughly $8^{\circ}$. At higher angles the lift curve's slope smoothly decreased until the maximum lift was achieved at approximately $14^{\circ}$, which was followed by a sudden decrease in lift. The maximum lift coefficient increased slightly from 1.15 at a Reynolds number of 60,000 to 1.20 at a Reynolds number of 300,000. The minimum drag, $C_{d} \approx 0.01$, occurred at $C_{l} \approx 0.25$ for Reynolds numbers of 200,000 and 300,000 . The minimum drag increased to $C_{d} \approx 0.02$ for $\operatorname{Re}=100,000$ and was fairly constant for $-0.3 \leq C_{l} \leq 0.7$, The minimum drag coefficient at $\mathrm{Re}=60,000$ was approximately 0.22 at a $C_{l}=-0.2$. The variation in the character of the drag polars indicate that this range of Reynolds numbers contains an interesting change in flow physics.

\section{Simulations}

A variety of test simulations were run using 120 panels to model the NACA 2414 airfoil, and were equally distributed over the top and bottom surfaces. This number is generally considered to be more than sufficient for an inviscid flow field calculation.

Example flow patterns are shown for 250 free vortices in Figure 3 and 500 free vortices in Figure 4. The simulations were done for airfoil at zero angle of attack and a Reynolds number of approximately 150,000. Both Figures show similar behaviour and as expected the distribution of free vorticity clusters in the 
wake of the airfoil. The results show that vortices shed from the lower surface are grouped to the lower half of the wake near the airfoil and diffuse across the wake as we move downstream.

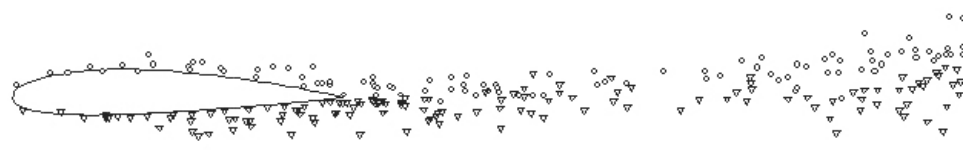

Figure 3: $\quad$ Vortex Cloud Model simulation of flow over a NACA 2414 airfoil at $\mathrm{Re}=149,503$, with 250 free vortices shed into the flow field.

A time step by time step examination of the motion of the shed vortices demonstrated a nearly uniform motion of the free vorticity in the far wake. One issue that was of concern is the number of vortices that needed to be shed to obtain an accurate representation of the flow field. Our results show similarity of the results for both 250 and 500 shed vortices demonstrates that a large enough number of vortices were being shed to obtain an accurate representation of the flow. All subsequent simulations were done using 500 shed vortices; however we believe that 250 should be sufficient.

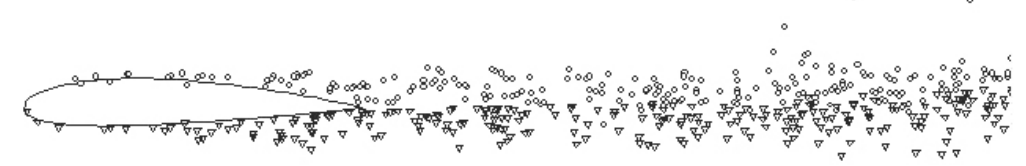

Figure 4: $\quad$ Vortex Cloud Model simulation of flow over a NACA 2414 airfoil at $\operatorname{Re}=149,503$, with 500 free vortices shed into the flow field.

Simulations of flow over the NACA 2414 airfoil were made for a range of angles of attack, $\alpha$, and are shown in Figure 5. The airfoil is displayed in its horizontal orientation and the free stream velocity is inclined upward as $\alpha$ is increased. We would expect that the wake would deflect downward for $\alpha=0^{\circ}$ as is clearly shown. There was no indication of flow separation over the airfoil at this low angle of attack. When $\alpha=5^{\circ}$, the wake deflected slightly upward in the direction of the free stream velocity, and as with the case of $\alpha=0^{\circ}$ no separation was evident. These angles of attack are within the linear portion of the lift curve and the flow would not be expected to have significant separation regions. As we move the nonlinear portion of the lift curve, near the maximum lift region of the lift curve, say $\alpha=10^{\circ}$, we would expect to see the presence of a significant portion of separated flow on the suction surface near the trailing edge. The simulation represents this situation reasonably well, with a separation zone that is approximately $40 \%$ of the chord length. Finally, the flow over the airfoil in the deep stall range, represented by $\alpha=15^{\circ}$, clearly shows a separated zone that extends over nearly the entire suction surface, approximately $80-90 \%$ of the chord length. In general, the qualitative picture is that the predicted flow appears to give a good qualitative representation of the actual flow. 


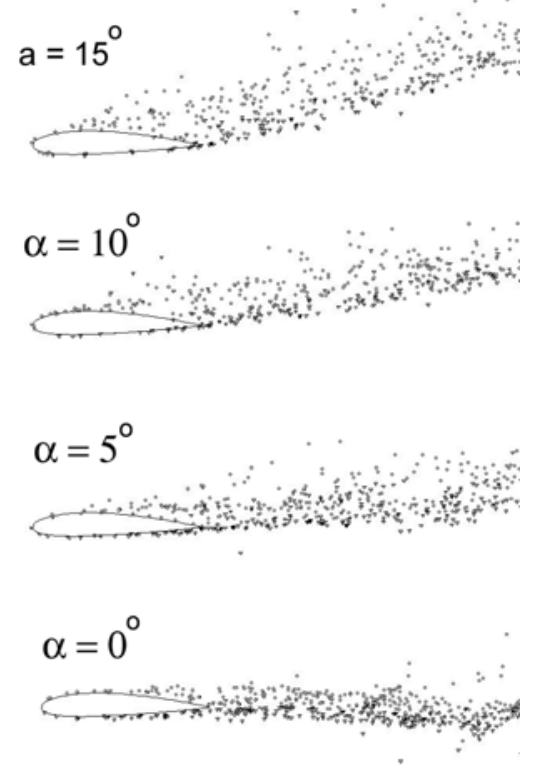

Figure 5: $\quad$ Vortex Cloud Model simulation of flow over a NACA 2414 airfoil at $\operatorname{Re}=149,503,500$ vortices shed, for $\alpha=0^{\circ}, 5^{\circ}, 10^{\circ}$ and $15^{\circ}$.

\section{Discussion}

Our assessment is that the vortex cloud model is capable of simulating the flow over an airfoil at low-Reynolds number. However we are concerned that a reliable means of estimating the actual forces on the airfoil has not been established. The likely cause of this could come from a number of sources. First, this is a random process that requires averaging and may require a large number of time steps to reach their asymptotic values. Second, the integration method was based on a simple trapezoid rule where a more accurate method may be required. However it should be noted that the method does work for a strictly inviscid estimate. Finally, we have to consider the possibility that the accuracy of the predicted, instantaneous, surface pressure distribution is not sufficiently accurate to allow us to compute these quantities accurately. A better practice may be to use a control volume approach to estimate these forces, such as the Betz and Jones wake traverse methods [8].

Several issues with the Vortex Cloud Model still need to be resolved. The vortex shedding method that was recommended by Lewis did not work as described. In that form the vorticity was wildly flung around the flow field, necessitating a $75 \%$ reduction of shed vortex strength for the method to function, indicating that a more formal scientifically based method determination of the shed vorticity needs to be established. As accurate drag measurements were not 
available we could not assess the validity of the random vortex displacement model.

Our observation on the application of the Vortex Cloud Model is that it has some significant advantages as a flow simulation tool. First, it is a grid free simulation which saves a lot of work. This is particularly important if it were to be used for the design of optimum shape airfoils. The optimization process typically requires the simulation of a large number of candidate airfoils, and benefits greatly if simulation effort is reduced. We found the method simple to apply and relatively fast, making it quite competitive with other methods.

\section{Conclusions}

The results of this work show that the vortex cloud method shows promise as a useful tool for airfoil simulation. The method is fairly easy to implement and is relatively fast. However, several issues, such as the calculation of the aerodynamic forces, the vortex shedding process, and suitability of the random displacement model need further consideration.

\section{Acknowledgements}

The authors would like to acknowledge the generous support of this work by the Natural Sciences and Engineering Research Council of Canada and E.H. Price. Ltd.

\section{References}

[1] Chorin, A.J., Numerical Study of Slightly Viscous Flow, Journal of Fluid Mechanics, 57, pp. 785-96, 1973.

[2] Chorin, A.J., Vortex Sheet Approximation of Boundary Layers, Journal of Computational Physics, 27, pp. 428-442, 1978.

[3] Lewis, R.I., Vortex Element Methods for Fluid Dynamic Analysis of Engineering Systems, Cambridge University Press, Cambridge, 1991.

[4] Selig, M.S., Donovan, J.F., and Fraser, D.B., Airfoils at Low Speeds, Soartech 8, Soartech Publications, Virginia Beach, U.S.A, 1989.

[5] Selig, M.S., Guglielmo, J.J., Broeren, A.P. and Giguere, P., Summary of Low Speeds Airfoil Data, Volume 1, Soartech Publications, Virginia Beach, U.S.A., 1995.

[6] Selig, M.S., Lyon, C.A., Giguere, P., Ninham, C.P. and Guglielmo, J.J., Summary of Low Speeds Airfoil Data, Volume 2, Soartech Publications, Virginia Beach, U.S.A., 1996.

[7] Lyon, C.A., Broeren, A.P, Giguere, P., Gopalarathnam, A. and Selig, M.S., Summary of Low Speeds Airfoil Data, Volume 3, Soartech Publications, Virginia Beach, U.S.A., 1997.

[8] Schlichting, H., Boundary-Layer Theory, McGraw-Hill, New York, pp. 758-777, 1979. 